\title{
Multi-modal / Multi-resolution Neural Circuit Reconstruction with Automated Tape Ultramicrotomy (ATUM) and Serial Section Scanning Electron Microscopy
}

\author{
Karl Friedrichsen, Pratyush Ramakrishna, Jen Chun Hsiang, Daniel Kerschensteiner and Josh Morgan
}

Washington University in St. Louis, St. Louis, Missouri, United States

Automated tape collection of ultrathin sections was developed to facilitate the collection of large numbers of sections (Hayworth et al., 2014; Schalek et al., 2012). Scanning electron microscopy of ultrathin sections preserved on the stable substrate of collection tape and silicon wafers also lends itself to the reimaging of tissue at multiple resolutions. Multi-resolution serial section SEM can be used to link 3D ultrastructure of local circuitry to millimeter-scale axon projections without the need to acquire petabytes of data. Micron-resolution mapping of 3D image volumes can also be readily correlated to 3D optical image volumes, adding functional or molecular information to electron microscopic circuit reconstructions.

We use this multi-modal / multi-resolution approach to uncover the synaptic connectivity of mouse retinal amacrine neurons that have been labeled transgenically and functionally characterized with two-photon calcium imaging. Subtype specific promotors were used to transgenically drive the expression of fluorescent proteins in mouse retinal amacrine cells. Light responses to amacrine neurons expressing the calcium indicator GCamp6 were characterized using two-photon live imaging. Retinas were then aldehyde fixed and reimaged with confocal microscopy to generate tissue maps of fluorescent protein expressing cell bodies relative to surrounding cell bodies and blood vessels. Cell bodies and blood vessels could be identified using aldehyde background fluorescence. Additional tissue reference signal could be generated by labeling nuclei with DAPI and enhancing background signal with Sulfarhodamine. After optical mapping, tissue was fixed in additional glutaraldehyde and stained using double layered osmium, uranyl acetate and lead aspartate. Retina tissue blocks ( $>1 \mathrm{~mm}^{\wedge} 2$ ) were cut into $\sim 1500$ ultrathin en face sections and mounted onto silicon wafers.

A Merlin scanning electron microscope and custom acquisition software (Wafermaper, (Hayworth et al., 2014) were used to map the full tissue sections at multiple resolutions. Initially, an EM montage image of each four-inch-wide wafer is acquired for identifying the position of sections. Three-millimeter-wide micrometer resolution images of each section are then acquired and aligned for targeting imaging to tissue subregions. Twenty nanometer resolution images are then targeted to regions that were optically mapped. Comparison of these medium resolution images to the fixed optical maps were used to identify transgenically labeled neurons within the EM tissue. High resolution, four nanometer pixel size, 3D image volumes were then acquired of the circuitry surrounding selected labeled neurons. After reconstruction of the high-resolution circuitry, an additional fast, ten nanometer resolution image volume was acquired to allow for the reconstruction of large neurites of interest identified within the high-resolution volume.

The ease of repeatedly imaging digitally mapped sections mounted on a solid substrate provides for relatively efficient, targeted and flexible exploration of datasets that would otherwise require many petabytes of storage to digitize in full.

\section{References}

Hayworth, K.J., Morgan, J.L., Schalek, R., Berger, D.R., Hildebrand, D.G.C., and Lichtman, J.W. (2014). Imaging ATUM ultrathin section libraries with WaferMapper: A multi-scale approach to EM reconstruction of neural circuits. Front. Neural Circuits 8 . 
Schalek, R., Hayworth, K., Kasthuri, N., Morgan, J.L., Berger, D., Wilson, A.M., Anger, P., Aderhold, D., Seung, H.S., and Lichtman, J.W. (2012). ATUM-based SEM for High-Speed Large-Volume Biological Reconstructions. Microsc. Microanal. 18. 